\title{
A Pediatric Case of Pityriasis Rubra Pilaris Successfully Treated with Low-Dose Vitamin A
}

\author{
Monji Koga ${ }^{a}$ Kaori Koga $a^{b} \quad J u i c h i r o ~ N a k a y a m a{ }^{a}$ \\ ${ }^{a}$ Department of Dermatology, Faculty of Medicine, and ${ }^{b}$ Department of \\ Pathology, Fukuoka University Hospital, Fukuoka, Japan
}

\section{Key Words}

Pityriasis rubra pilaris · Vitamin A · Etretinate $\cdot$ Adverse effect $\cdot$ Pediatric case

\begin{abstract}
Pityriasis rubra pilaris (PRP) is a rare chronic inflammatory keratosis that is clinically characterized by gradually developing reddish or orange extending plaques and keratotic follicular papules. In pediatric patients, we frequently hesitate to administer certain medications for treatment of PRP, specifically etretinate, systemic corticosteroids, and biologics recommended by previous studies. Although administration of high-dose vitamin A was described in a previous textbook of dermatology, details about the lower limits and treatment periods were not provided. We presented a pediatric case of PRP that was successfully controlled with minimum dosage of systemic vitamin $A$ in the literature. Before and 14 days after beginning the therapy, both vitamin A levels of peripheral blood were within the normal range. We considered that the clinical efficacy may not be due to a supplementary effect of vitamin $A$, but to a pharmacological action because serum vitamin $A$ was within the normal limits during the therapy.
\end{abstract}

\section{Introduction}

Pityriasis rubra pilaris (PRP) is a rare chronic inflammatory keratosis, clinically characterized by gradually developing to reddish or orange extending plaques and keratotic follicular papules.

Five types of PRP were classified by Griffith [1], and there are refractory cases (particularly in type II) in contrast with spontaneous cures for several years as described in the literature. At the onset in infancy, we frequently hesitate to use these medications, which are etretinate, systemic corticosteroid, and biologics recommended 
by previous studies. An administration of high-dosage vitamin A was described in the previous textbook of dermatology [2]; however, it did not describe in detail the lower limits and the period. Herein, we present an infantile case of PRP which was hard to classify, successfully controlled with a minimal dose of systemic vitamin A in the long term.

\section{Case Report}

A 12-year-old male patient (weight $40 \mathrm{~kg}$ ) first presented to our hospital because of the presence of reddish asymptomatic keratosis plaques with no medications in January 2008. The patient was not taking any medications to date (including s-retinoids, albumin, etc.). The plaques had appeared on the palms and plantar aspects of the feet 6 years previously. He had no familial history of inflammatory dermatitis or allergic disorders. Erythematous plaques and keratotic follicular papules emerged and rapidly expanded to cover the head, trunk, and extremities over the past year (fig, 1a-c).

Histopathologically, a biopsy specimen obtained from a reddish plaque on the patient's back showed hyperkeratosis, acanthosis, and elongation of rete ridges in the epidermis. Alternating vertical and horizontal parakeratosis in the epidermis (checkerboard appearance) with follicular keratinization was also observed ( $\underline{\text { fig. } 2 \mathrm{a}}$, b). These typical findings resulted in a final diagnosis of PRP. Although topical betamethasone butyrate propionate, maxacalcitol, and oral loratadine at 10 $\mathrm{mg}$ /day were introduced in February 2008, the skin lesions rapidly expanded and merged into larger plaques within 2 months, eventually covering the whole trunk (fig. 1d).

Although we recommended oral etretinate according to the literature at first, his family disagreed with our suggestion. One month later, we recommended to take low-dose vitamin A and started vitamin $A$ in oil $\left(\mathrm{Chocola}^{\circledast}\right)$ at 10,000 units twice a day in addition to the above-mentioned agents Before and 14 days after beginning the therapy, both vitamin A levels of peripheral blood were within the normal range, and no liver or renal dysfunction were seen. Approximately 2 months after starting the vitamin A, the reddish eruptions on the whole body, including those on the palms and plantar aspects of the feet, had essentially disappeared (fig. 1e). Therefore, we discontinued the vitamin A in oil, but the eruptions on the trunk gradually relapsed 2 weeks after discontinuation. Since then, we have regularly followed up the patient and have prescribed the agent during rash exacerbations.

\section{Discussion}

Most pediatric cases of PRP classified as type III by Griffith [1] generally undergo spontaneous cure between 1 and 3 years. Although the present case was a suspected type III with no family history and a juvenile onset, the 6-year history of rash on the palms and plantar aspects of the feet may have been associated with PRP; therefore, we finally could not establish a precise classification. Although the possibility of spontaneous healing was not denied in the present case, we stopped the administration of vitamin A after the change in the patient's eruption; the rashes were worsening and expanding again in short time. Soon after re-administration of the same dose, the rashes immediately improved, indicating that the amount of the vitamin A was effective for the dosing period.

Administration of large amounts of vitamin A for treatment of PRP has been described in previous literature and textbooks [2, 3], but the effective amount and period of vitamin A has not been specifically described.

Specifically, the previous dosage was 1,000,000 units per day for 14 days [4] and 200,000 units per day for 35 days [5]. Both cases showed immediate improvement, but 
relapsed after a while. Therefore, re-administration of the same dose was performed, and immediate efficacy was again obtained. In the present case, we made a decision to administer 10,000 units twice a day based on the recommendations for over-thecounter drug vitamin A in Japan, which define the safe amount as 5,000 units per day continuously. The amount in the present case was the minimum effective dosage of vitamin A in the previous literature. These results suggested that the clinical efficacy may not be due to a supplementary effect of vitamin A, but to a pharmacological action because serum vitamin A was within the normal limits during the therapy.

However, there are few similar case reports to date. Hereafter, as more cases become accumulated, we would expect to clarify the effective minimum dosage, optimal dosing period, and adverse effects of vitamin A therapy for pediatric PRP patients.

\section{Disclosure Statement}

The author declare that they have no conflict or interest
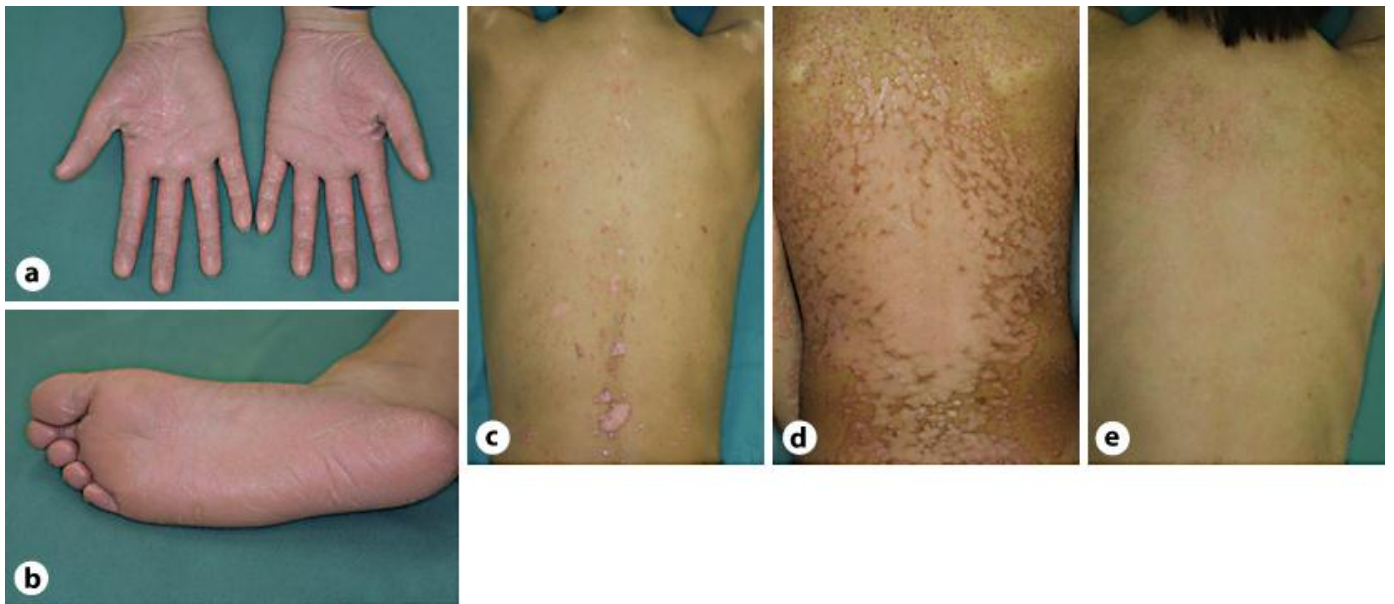

Fig. 1. a-c Before administration of systemic vitamin A, clinical manifestations were generalized erythroderma, a large number of reddish erythematous plaques and keratotic follicular papules on the limbs and trunk, and palmoplantar keratoderma. $\mathbf{d}$ The skin lesions rapidly expanded and merged into larger plaques within 2 months. e After 1 month of therapy with oral vitamin A, the rash significantly improved with only slight residual erythema. 


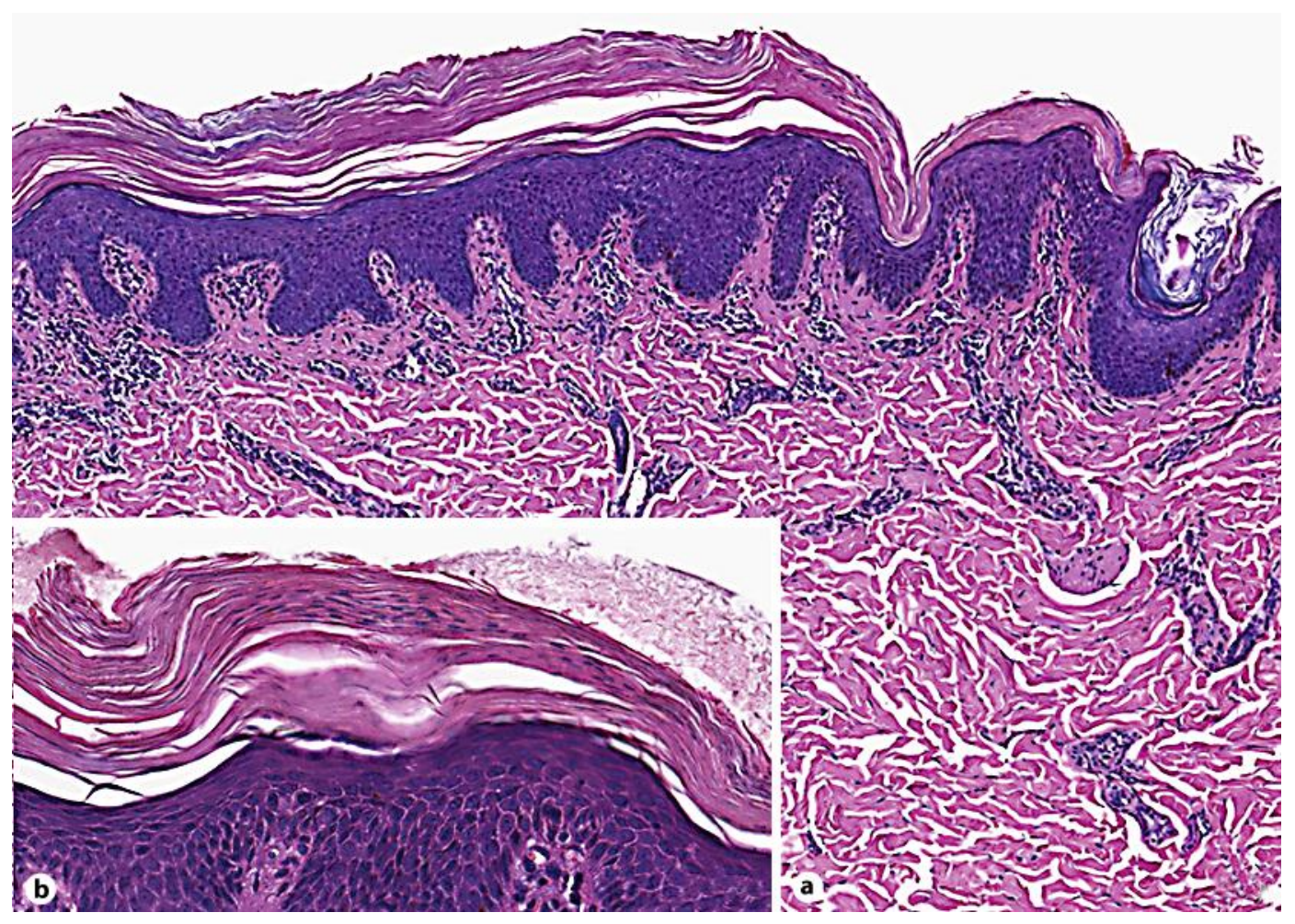

Fig. 2. a Hyperkeratosis with keratin plugging and prolonged rete ridges were observed with mild perivascular lymphocytic infiltration (hematoxylin-eosin stain, original magnification $\times 40$ ). b A checkerboard appearance was also seen (hematoxylin-eosin stain, original magnification $\times 200$ ).

\section{References}

1 Griffiths WA: Pityriasis rubra pilaris. Clin Exp Dermatol 1980;5:105-112.

2 Lowell AG, Howard PB: Pityriasis Rubra Pilaris. Fitzpatrick's DERMATOLOGY, 6th edition, pp 442-444.

-3 Logan WS: Vitamin A and keratinization. Arch Dermatol 1972;105:748-753.

-4 van Voorst Vader PC, van Oostveen F, Houthoff HJ, Marrink J: Pityriasis rubra pilaris, vitamin A and retinol-binding protein: a case study. Acta Derm Venereol 1984;64:430-432.

5 Kellum RE: Possible significance of aqueous emulsified vitamin A in effective therapy for pityriasis rubra pilaris. J Am Acad Dermatol 1989;20:126-128. 